\title{
Interactive comment on "Distributed summer air temperatures across mountain glaciers: climatic sensitivity and glacier size" by Thomas E. Shaw et al.
}

\section{Anonymous Referee \#1}

Received and published: 27 October 2020

Shaw and others present an analysis of air temperatures observed over the surfaces of several mountain glaciers on the Tibetan Plateau. The study follows similar work conducted in western Canada (Shea and Moore, 2010), the Italian Alps (Carturan and others, 2015), and Chile (Ayala and others, 2015), which demonstrates that air temperatures observed over the surface of melting glaciers are predictably different from off-glacier 'ambient' temperatures that would be expected at the same elevation. Flow length appears to play a control on air temperatures, and the authors investigate their findings from the Parlung cathment in the context of a number of similar studies and datasets. Overall the paper is well-written, though the language could be simplified in many places to reduce the manuscript length and improve readability. 
General comments: - As the paper is mostly focused on the datasets from the Tibetan Plateau, the title is bit misleading. Yes, there are some comparisons made with other datasets, but the title implies a global scale analysis.

- Sec. 4.3; The methods for analyzing the 'global' temperature datasets given in Table 2 are well-motivated, but questionable. An assumption of a constant $6.5 \mathrm{C} / \mathrm{km}$ lapse rate will likely result in substantial errors in the estimated ambient air temperatures, which will then skew the values calculated for $\mathrm{k} 1$ and $\mathrm{k} 2$. I think the approach here needs to be improved before such comparisons can be made.

- The parameter k2 is termed "climatic sensitivity", but this is also a bit confusing. I would interpret climatic sensitivity as being related to changes in climate (e.g. $\mathrm{mm}$ w.e. /C). Specifically, $\mathrm{k} 2$ is unitless and it defines the relation between Ta_glac and Ta_amb during katabatic flows, so it really just quantifies the "cooling effect" a glacier has on the air mass immediately above it ( $1=$ no cooling, $0.10=$ strong cooling). My suggestion would be to use a different term, though I'm not sure "cooling effect" is the right one.

Specific comments: - L26: remove "several, "

- L26: see comments on "climate sensitivity"

- L30: "slower decrease of climate sensitivity" is tough to parse. How about "Beyond this distance. . .glacier datasets show little additional cooling effects."

- L31-32: It's not the observations that are sensitive, it's the glaciers themselves: "In general, small glaciers. . .have little cooling effect and are thus highly sensitive to changes..."

- L37: the "beyond" here seems to imply past or in front of the glacier. Suggest rephrasing.

- L47: suggest ". . .the use of linear temperature gradients, typically..."

- L48-53: suggest shortening and simplifying: "A free-air ELR cannot be reliably used to

\section{(1)}


estimate near-surface air temperatures above melting glaciers, where steep gradients are found within $10 \mathrm{~m}$ of the surface (REFS)."

- L55: clarify that the overestimation occurs in energy balance models.

- L65-69: This is important, and l'd suggest moving it upwards as a standalone paragraph that also highlights that there are two main models for melt estimation.

- L73: "western Canada" or "southern Coast Mountains", not Canadian Rockies.

- L83-84: for clarity, remove "based upon. ..(1998)" - Gruell and Bohm is described in the next sentence

- L88: remove ",however,"

- L95: "The ModGB..."

- L103: “...though it does not explicitly account for physical processes that would reduce the glacier cooling effect at the glacier terminus."

- L106: see general comments above

- L113: can you stick with 'transferrability'? "generalizability" is a mouthful, and not commonly used in this field

- L113-L114: "As such, the transferability of near-surface air temperature models remain largely unknown. Analysis ..."

- L115-116: simplify: "In this study, we use new datasets of on-glacier air temperatures observed at three glaciers ..."

- L118: the word 'distributed' is probably unneeded

- Sec 3.3 can be moved up and included with the 3.1, which can be renamed "Meteorological observations", but just start with "Air temperatures (Ta), incoming shortwave,..."

- L193-194: remove the first part: "Flowline distances $(m)$ for each glacier were calcu- 
lated with the TopoToolbox..."

- L200: remove "between the aforementioned studies."

- L206-207: Feels like this needs a more general introduction: "Our methods consist of (1) aggregating temperature observations based on off-glacier temperatures, (2) generating off-glacier temperature gradients to compare on and off-glacier temperatures, and (3) estimating the glacier cooling effect on near-surface temperatures by fitting parameters to the SM10 model." Your rationale can then be moved into the individual subsections.

- L226: replace "within the glacier boundary layer" with "between ambient and onglacier temperatures"

- L226 - 229: this could be simplified to read "We calculate hourly ambient lapse rates from the off-glacier weather station and temperature loggers T1_94, T2_94, and T1_390. These sites are assumed to be unaffected by the glacier cooling effect."

- L234: “... T-logger to quantify the difference between ambient and on-glacier Ta at each site..."

- Eq 1: asterisks should be superscript in this equation, and see general comments above

- L276: suggest using 'transferability' instead of 'generalisability'

- L284: is the ELR suitable for all sites and seasons?

- L308: since there is a scale break in the figure, it is tough to see this as 'linear'

- L349 - 351: Simplify! "For P90 conditions (Fig 6a), differences between TaAMB and observed on-glacier temperatures are up to $5.8 \mathrm{C}$ at flowline distances greater than 7000 m."

- L351: avoid 'heighten' in this context 
- Sec. 5.2 : avoid using "offset"

- Figure 7: perhaps show these relations as scatterplots. Tough to interpret the relation using line and bar plots.

- L352 - 353: RH will typically be high when temperatures are low, so this is not surprising.

- L382: "notably distinct" doesn't really fit with the results in the table, as the coefficients of SM10 are within the confidence intervals of the other datasets

- L430: need to specify energy balance models. Temperature indexed approaches don't need this correction if the melt factors are calculated with off-glacier temperatures.

- L474: "While the data from the Parlung catchment. .."

- L493: southern Coast Mountains (not Canadian Rockies)

Interactive comment on The Cryosphere Discuss., https://doi.org/10.5194/tc-2020-198, 2020. 